\section{Impact of Different Catheter Lock Strategies on Bacterial Colonization of Permanent Central Venous Hemodialysis Catheters}

\author{
Stefan Erb, MD $;^{1}$ Andreas F. Widmer, MD, MS $;^{1}$ \\ Sarah Tschudin-Sutter, $\mathrm{MD} ;^{1}$ Ursula Neff, $\mathrm{RN}$; $^{\mathbf{1}}$ \\ Manuela Fischer, $\mathrm{RN}^{2}$ Michael Dickenmann, $\mathrm{MD}^{2}$ \\ Philipp Grosse, $\mathrm{MD}^{2}$
}

\begin{abstract}
Thirty-nine hemodialysis patients with permanent central venous catheters were analyzed for bacterial catheter colonization comparing different catheter-lock strategies. The closed needleless Tego connector with sodium chloride lock solution was significantly more frequently colonized with bacteria than the standard catheter caps with antimicrobially active citrate lock solution (odds ratio, 0.22 [95\% confidence interval, 0.07-0.71]; $P=.011$ ).
\end{abstract}

Infect Control Hosp Epidemiol 2013;34(12):1314-1317

Catheter-related bloodstream infection (CRBSI) is a serious complication in patients with tunneled long-term central venous catheters (CVCs) undergoing chronic hemodialysis. ${ }^{1}$ Proper aseptic technique when manipulating CVCs or the use of antimicrobial lock solutions is recommended to reduce the incidence of CRBSIs. ${ }^{2}$ The impact of closed needleless devices to prevent CRBSI is controversial. ${ }^{3}$ The Tego system by ICU Medical is a needleless luer lock connector device for hemodialysis CVCs that is approved by the US Food and Drug Administration. This connector is locked with saline solution and remains in place for 3 consecutive hemodialysis sessions, reducing manipulation of the catheter hub, an important source of intraluminal colonization of long-term CVCs and subsequent CRBSI. ${ }^{4,5}$

In August 2008, the Tego connector system was introduced in all patients undergoing hemodialysis over a permanent tunneled CVC at the dialysis unit at the University Hospital Basel, a tertiary teaching hospital in Switzerland. Within routine surveillance after introduction of the Tego system, an increase of positive blood cultures drawn from the hemodialysis CVC was detected.

Subsequently, reports of high rates of CRBSI associated with the use of closed needleless mechanical valve systems were published. ${ }^{3}$ We therefore performed a sequential observational study from 2010 until 2012 to determine the colonization rate of permanent tunneled hemodialysis CVCs with different catheter connector (Tego vs conventional closing caps [CCCs]) and lock solution (sodium chloride vs citrate) strategies.

\section{METHODS}

Study Setting, CVC Locking Techniques, and Infection Control Activities

From August 2008 to June 2010, the Tego closed needleless connector device system (ICU Medical) was used for all hemodialysis CVCs, according to the manufacturers' instructions: disinfection of the connector injection site with ethanol swabs $80 \%(\mathrm{v} / \mathrm{v})$ before and after each use, locking of each catheter branch with $2 \mathrm{~mL}$ of sterile $0.9 \%$ sodium chloride between hemodialysis sessions, and changing the Tego every 7 days before the fourth dialysis. ${ }^{4}$ In July 2010, the Tego devices of all hemodialysis CVCs were replaced with CCCs (Discofix closing cap, Braun). CCC catheter hubs were disinfected with octenidine-containing solutions before attachment and after detachment of a device. Catheter branches were blocked with $2 \mathrm{~mL}$ of citrate lock solution: $46.7 \%$ citrate until April 2011 and 30\% citrate thereafter to reduce the risk of dangerous hypocalcemias in case of accidental citrate flushing. CCCs were changed after each dialysis session (Table 1).

Standard infection control protocols were used with all connector systems when manipulating dialysis catheters. ${ }^{2} \mathrm{An}$ additional infection prevention program was implemented in the dialysis unit from August 2010 until April 2011 (during this period, CCCs $46.7 \%$ citrate was in use) and included the following interventions: additional hand disinfectant dispensers were installed to facilitate hand hygiene, improved $\mathrm{CVC}$ care sets were implemented for catheter manipulation, and teaching about aseptic catheter management for healthcare workers was provided.

\section{Microbiological Culture of Catheter Lock Solutions}

Catheter lock solutions were examined for bacterial growth at 3 serial time points using 3 different locking strategies (Table 1): (1) Tego needleless connector device with $0.9 \%$ sodium chloride lock solution (June 28-29, 2010), (2) CCCs with $46.7 \%$ citrate lock solution (August 9-10, 2010) before infection prevention program, and (3) CCCs with $30 \%$ citrate lock solution after completion of the infection prevention program (June 6-7, 2011). Five milliliters of blood, including the lock solution from the arterial and the venous branch, was aseptically drawn in aerobic blood culture bottles (BacT/ ALERT) and incubated for 5 days at $37^{\circ} \mathrm{C}$. Species identification of positive cultures was done using the Vitek system (bioMérieux).

Colonization was defined as any positive culture of a lock solution. Colonized CVCs were not treated with systemic antibiotic or antibiotic lock. 
TABLE 1. Baseline Characteristics and Results of Tego $0.9 \%$ Sodium Chloride, Conventional Closing Caps (CCCs) $46.7 \%$ Citrate, and CCCs $30 \%$ Citrate Groups

\begin{tabular}{|c|c|c|c|}
\hline & Tego $0.9 \%$ sodium chloride & CCCs $46.7 \%$ citrate before IPP & CCCs $30 \%$ citrate after IPP \\
\hline Age, median (range), years & $68(29-87)$ & $67(46-87)$ & $68(46-89)$ \\
\hline Time in use & August 15,2008 -July 5,2010 & July 6, 2010-March 31,2011 & April 1, 2011-June 1, 2012 \\
\hline No. of catheter-days ${ }^{\mathrm{a}}$ & 12,512 & 6,994 & 12,840 \\
\hline Date of lock solution culture & June 26-July 5, 2010 & August $9-10,2010$ & June 6-7, 2011 \\
\hline Exchange of device & Before fourth dialysis & After each dialysis & After each dialysis \\
\hline Catheter colonization (\%) & $16(52)$ & $5(19)^{b}$ & $2(7)^{\mathfrak{c}}$ \\
\hline No. of microorganisms isolated & 19 & 7 & 2 \\
\hline Microorganisms frequency & $\begin{array}{l}17 \text { CoNS, } 1 \text { Streptococcus san- } \\
\text { guinis, } 1 \text { Micrococcus }\end{array}$ & $\begin{array}{l}4 \text { CoNS, } 1 \text { Enterococcus faecalis, } \\
1 \text { Enterobacter cloacae, } 1 \text { Pseu- } \\
\text { domonas aeruginosa }\end{array}$ & 1 CoNS, 1 E. faecalis \\
\hline
\end{tabular}

моте. Infection prevention program (IPP) from September 2010 to April 2011. CI, confidence interval; CoNS, coagulase-negative staphylococci; $O R$, odds ratio.

a Catheter-days were calculated for each group for the period during which the connector lock solution system was in use.

b Between Tego and CCCs $46.7 \%$ citrate: OR, 0.22 (95\% CI, 0.07-0.71); $P=.011$.

c Between Tego and CCCs 30\% citrate: OR, 0.07 (95\% CI, 0.01-0.35); $P=.001$.

\section{Statistical Analysis}

We performed logistic generalized estimating equations modeling, using the lock strategies as the covariate and the presence of colonization as the outcome. On the basis of the generalized estimating equations, we derived odds ratios (ORs) with corresponding 95\% confidence intervals (CIs). Values of $P<.05$ were considered statistically significant.

\section{RESULTS}

During the study period, a total of 39 patients received hemodialysis by permanent tunneled, double-lumen CVC. Thirty-one patients had the Tego connector system at the first screening date in June 2010. After changing the Tego system to the CCCs system with $46.7 \%$ citrate lock solution, 26 patients could be screened in August 2010 before the infection prevention program started. Five patients of the initial population could not be analyzed because of catheter removal. The third screening was done in 30 patients with CCCs and $30 \%$ citrate lock in June 2011 after completion of the infection prevention program (Table 1).

In the Tego group, 16 of 31 patients (52\%) had bacterial growth of at least 1 microorganism from the sodium chloride lock solution. In comparison, the colonization rate of the patients with the CCCs and citrate lock was significantly lower: in the CCCs group with $46.7 \%$ citrate lock, only 5 of $26(19 \%)$ patients had positive cultures (OR, $0.22[95 \% \mathrm{CI}$, $0.07-0.71$ ]; $P=.011$ ); in the CCCs group with $30 \%$ citrate lock, 2 of $30(7 \%)$ catheters were tested positive (OR, 0.07 [95\% CI, 0.01-0.35]; $P=.001$; Figure 1).
Nineteen patients underwent all 3 screenings without intercurrent catheter replacements. The colonization rate between the 3 groups was comparable to the above mentioned (data not shown).

Coagulase-negative staphylococci were the most common pathogens detected in all 3 groups (Tego, $89 \%$; CCCs $46.7 \%$ citrate, $75 \%$; CCCs $30 \%$ citrate, $50 \%$; Table 1 ). The number of CRBSIs during the study period was analyzed retrospectively and showed no significant difference between the 3 groups (CRBSIs per 1,000 catheter-days: Tego, 0.24; CCCs $46.7 \%$ citrate, 0.28 ; CCCs $30 \%$ citrate, 0.16 ).

\section{DISCUSSION}

In our study, we found a significantly higher colonization rate of more than $50 \%$ of the saline lock solution in patients with the Tego needleless connector device compared with the $46.7 \%$ and $30 \%$ citrate lock solutions with CCCs. Several reasons may explain our findings. The antimicrobial potency of the lock solutions might have a considerable impact on catheter colonization. With the Tego connector, the manufacturer recommends $0.9 \%$ sodium chloride as the lock solution, which does not prevent bacterial growth. In contrast, citrate has antimicrobial activity and has been advocated for hemodialysis CVC locking. ${ }^{6,7}$ It would be interesting to use citrate lock solution in conjunction with the Tego device; however, to date, this procedure is not supported by the manufacturer.

The implementation of an active surveillance program at the dialysis unit-including microbiological examination of 


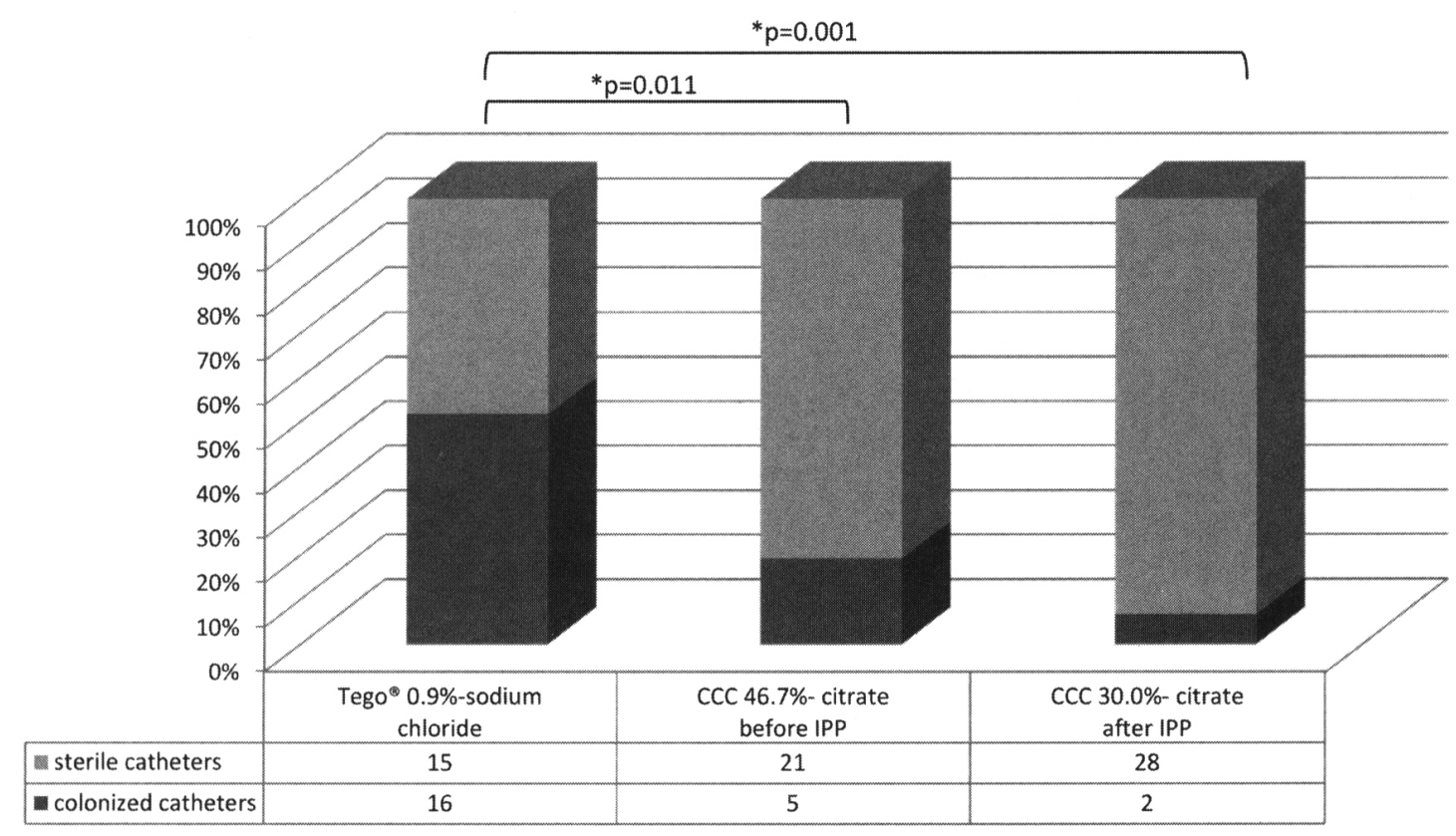

FIGURE 1. Percentage of colonized central venous catheters (CVCs) with the 3 catheter lock strategies of all 39 patients. Absolute numbers and percentages of sterile and colonized CVCs are indicated. Values of $P<.05$ were considered statistically significant. CCC, conventional closing cap; IPP, infection prevention program.

the catheter lock solution and the change from the Tego sodium chloride to the CCCs $46.7 \%$ citrate lock system-could have lead to improved hygienic catheter management with consequently lower CVC colonization rates with the CCCs. Reduction of nosocomial infections by surveillance has been demonstrated by the Study on the Efficacy of Nosocomial Infection Control (SENIC). ${ }^{8}$ The infection prevention program, which was implemented after microbiological screening of the $46.7 \%$ citrate lock solution, might explain the lower colonization rate of the $30 \%$ citrate lock solutions, emphasizing the importance of surveillance and infection prevention programs to reduce CRBSI. ${ }^{9}$ However, statistical analysis between the CCCs $46.7 \%$ and $30 \%$ citrate group was not performed because of model overfit due to the low number of colonized catheters.

Previous studies showed a clear correlation between catheter colonization and CRBSI. ${ }^{10}$ Although we found a significantly lower colonization rate of the CCCs citrate compared with the Tego sodium chloride lock system, we could not demonstrate a reduction of CRBSIs. However, our study was not designed to detect CRBSI; data about CRBSI were collected retrospectively, and the CRBSI rate was very low in all 3 groups (overall, 0.21 CRBSIs per 1,000 catheter-days).

In conclusion, infection prevention programs and an antimicrobial active lock solution such as citrate may considerably reduce the bacterial colonization rate of permanent hemodialysis CVC. The ease of use of closed needleless connection devices from the Tego type without an antimicrobial active lock should be balanced against the risk of infections, since these are usually preceded by colonization.

\section{ACKNOWLEDGMENTS}

We would like to thank PD Dr. med. M. Koller (Division of Clinical Epidemiology, University Hospital Basel) and Marc Dangel, MPH (Division of Infectious Diseases and Hospital Epidemiology, University Hospital Basel), for revising the statistical analysis of our data.

Potential conflicts of interest. All authors report no conflicts of interest relevant to this article. All authors submitted the ICMJE Form for Disclosure of Potential Conflicts of Interest, and the conflicts that the editors consider relevant to this article are disclosed here.

Affiliations: 1. Division of Infectious Diseases and Hospital Epidemiology, University Hospital of Basel, Switzerland; 2. Division of Nephrology and Transplantation Immunology, University Hospital of Basel, Switzerland.

Address correspondence to Andreas F. Widmer, MD, MS, Division of Infectious Diseases and Hospital Epidemiology, University Hospital Basel, Petersgraben 4, 4031 Basel, Switzerland (andreas.widmer@usb.ch).

Presented in part: 22nd European Congress of Clinical Microbiology and Infectious Diseases; London; 2012.

Received June 16, 2013; accepted August 23, 2013; electronically published October 28, 2013.

(C) 2013 by The Society for Healthcare Epidemiology of America. All rights reserved. 0899-823X/2013/3412-0012\$15.00. DOI: $10.1086 / 673987$

\section{REFERENCES}

1. Allon M, Daugirdas J, Depner TA, Greene T, Ornt D, Schwab SJ. Effect of change in vascular access on patient mortality in hemodialysis patients. Am J Kidney Dis 2006;47(3):469-477. 
2. O'Grady NP, Alexander M, Burns LA, et al. Guidelines for the prevention of intravascular catheter-related infections. Am J Infect Control 2011;39(suppl 1):S1-S34.

3. Salgado CD, Chinnes L, Paczesny TH, Cantey JR. Increased rate of catheter-related bloodstream infection associated with use of a needleless mechanical valve device at a long-term acute care hospital. Infect Control Hosp Epidemiol 2007;28(6):684-688.

4. ICU Medical. The Benefits of a Saline Flushing Protocol when using the Tego $($ Needlefree Hemodialysis Connector. San Clemente, CA: ICU Medical, 2012.http://www.icumed.com/media /95894/M1-1302_Saline_Flush_Recommendation_Tego.pdf.

5. McAfee N, Seidel K, Watkins S, Flynn JT. A continuous quality improvement project to decrease hemodialysis catheter infections in pediatric patients: use of a closed luer-lock access cap. Nephrol Nurs J 2010;37(5):541-545.

6. Yahav D, Rozen-Zvi B, Gafter-Gvili A, Leibovici L, Gafter U, Paul M. Antimicrobial lock solutions for the prevention of infections associated with intravascular catheters in patients un- dergoing hemodialysis: systematic review and meta-analysis of randomized, controlled trials. Clin Infect Dis 2008;47(1):83-93.

7. Weijmer MC, van den Dorpel MA, Van de Ven PJ, et al. Randomized, clinical trial comparison of trisodium citrate $30 \%$ and heparin as catheter-locking solution in hemodialysis patients. $J$ Am Soc Nephrol 2005;16(9):2769-2777.

8. Haley RW, Morgan WM, Culver DH, et al. Update from the SENIC project. Hospital infection control: recent progress and opportunities under prospective payment. Am J Infect Control 1985;13(3):97-108.

9. Patel $\mathrm{PR}$, Yi SH, Booth $\mathrm{S}$, et al. Bloodstream infection rates in outpatient hemodialysis facilities participating in a collaborative prevention effort: a quality improvement report. Am J Kidney Dis 2013;62(2):322-330.

10. Rodriguez-Aranda A, Alcazar JM, Sanz F, et al. Endoluminal colonization as a risk factor for coagulase-negative staphylococcal catheter-related bloodstream infections in haemodialysis patients. Nephrol Dial Transplant 2011;26(3):948-955. 